\title{
Angular dependence of nanoparticle generation in the matrix assembly cluster source
}

\author{
Maria Chiara Spadaro ${ }^{1, \dagger}$, Junlei Zhao ${ }^{2}$, William D. Terry ${ }^{3}$, Jian Liu $^{3,4}$, Feng Yin ${ }^{5}$, Flyura Djurabekova ${ }^{2}$, and Richard \\ E. Palmer ${ }^{1}(\Delta)$ \\ ${ }^{1}$ College of Engineering, Swansea University, Bay Campus, Fabian Way, Swansea SA1 8EN, UK \\ ${ }^{2}$ Department of Physics and Helsinki Institute of Physics, University of Helsinki, P. O. Box43, FIN-00014, Helsinki, Finland \\ ${ }^{3}$ Nanoscale Physics Research Laboratory, School of Physics and Astronomy, University of Birmingham, Birmingham B152TT, UK \\ ${ }^{4}$ National Center for Electron Microscopy in Beijing, Key Laboratory of Advanced Materials (MOE), The State Key Laboratory of New Ceramics and Fine \\ Processing, School of Materials Science and Engineering, Tsinghua University, Beijing 100084, China \\ ${ }^{5}$ School of Physics and Information Technology, Shaanxi Normal University, Xian 710062, China \\ ${ }^{\dagger}$ Present address: Catalan Institute of Nanoscience and Nanotechnology (ICN2), CSIC and BIST, Campus UAB, Bellaterra, 08193 Barcelona, Catalonia, \\ Spain
}

(C) The Author(s) 2019

Received: 8 August 2019 / Revised: 22 October 2019 / Accepted: 27 October 2019

\begin{abstract}
The matrix assembly cluster source (MACS) represents a bridge between conventional instruments for cluster beam deposition (CBD) and the level of industrial production. The method is based on $\mathrm{Ar}^{+}$ion sputtering of a pre-condensed $\mathrm{Ar}-\mathrm{M}$ matrix (where $\mathrm{M}$, is typically a metal such as $\mathrm{Ag}$ ). Each $\mathrm{Ar}^{+}$ion produces a collision cascade and thus the formation of metal clusters is in the matrix, which are then sputtered out. Here we present an experimental and computational investigation of the cluster emission process, specifically its dependence on the $\mathrm{Ar}^{+}$ion angle of incidence and the cluster emission angle. We find the incidence angle strongly influences the emerging cluster flux, which is assigned to the spatial location of the deposited primary ion energy relative to the cluster into the matrix. We also found an approximately constant angle between the incident ion beam and the peak in the emitted cluster distribution, with value between $99^{\circ}$ and $109^{\circ}$.
\end{abstract}

\section{KEYWORDS}

cluster beam deposition scale-up, nanoparticles, silver, ligand-free, green synthesis

\section{Introduction}

Cluster beam sources were developed in the early 1980s for fundamental studies of free cluster physics, leading, for example, to the discovery of magic numbers in metal and rare gas clusters $[1,2]$. Within 10 years, these instruments were adapted to enable cluster beam deposition (CBD) onto surfaces [3]. Such sources are based on physical processes for material vaporisation and then cluster (nanoparticles) aggregation, that highly differ from the chemicalbased approaches. CBD sources are characterized by a number of potential advantages, such as (i) purity, the nanoparticles are produced with a solvent-free synthesis route and, therefore, are characterized by high surface activity as no ligands or reducing agents are attached to their surfaces [4]; (ii) versatility, as the cluster can be produced from any base material (metal, alloy, semiconductor), while conventional chemical routes rely on the availability of different precursors [4-6]; (iii) tunable defects, as under controlled conditions it is possible to synthesize defect-rich material which can broad on their potential applications [7, 8]. Furthermore, by coupling the CBD source with the mass selection stage, it is possible to select the size of the clusters [9]. The cluster produced following this route are exploited in many diverse research fields, ranging from magnetism $[10,11]$ and plasmonics $[12,13]$ to catalysis $[4,14]$ and biomedicine [15].

The beam current from such CBD sources is typically limited to the 0.1-1 nanoampere regime. The amount of material generated is thus only $\sim \mu \mathrm{g} / \mathrm{h}[16]$, or below. Thus is generally sufficient for model studies, but cluster beam research also has the potential to contribute to technological challenges, e.g., in biomedicine or fine chemicals catalysis. To reach this goal, it is necessary to scale-up the deposition rate up to $\sim \mathrm{mg} / \mathrm{h}$ or even $\sim \mathrm{g} / \mathrm{h}$ rate.

A number of efforts to scale-up cluster beam deposition have been reported [17-20]. In this context, a completely new type of cluster beam source, the matrix assembly cluster source (MACS), was invented by our group [21-25]. In this approach, evaporated metal atoms are co-condensed with rare gas atoms on a substrate cooled down to $10-20 \mathrm{~K}$ in vacuum. An $\mathrm{Ar}^{+}$ion gun is then exploited to bombard the matrix. Metal clusters are produced, ripened and sputtered out of the matrix as a result of the collision cascades initiated by successive incident ions. The cold matrix acts as the refrigerant needed to assist body collisions [21]. Early applications of clusters from the MACS include catalysis of the oxygen evolution reaction (OER) over Co clusters in alkaline media [25] and the vapour phase selective hydrogenation of 1-pentyne to 1-pentene over $\mathrm{Pd}$ and $\mathrm{Pd} / \mathrm{Au}$ clusters [4]. Therefore, this novel cluster source can be used to produce any kind of nanostructured material, both single elements or alloys, with unprecedented deposition rates. Its development represent a serious step forward in the prospective exploitation of cluster properties in technological applications.

Understanding the cluster emission mechanism is fundamental to improve the MACS performance further. In this paper we present 
a systematic experimental study of the effect of the incident ion beam angle upon the cluster beam flux, and of the angular distribution of the clusters emerging. The experiments are complemented by large scale molecular dynamic simulations.

\section{Experimental and computational methods}

The configuration of the MACS employed in the experiments is shown elsewhere $[22,24]$ and the incident and collection angles are defined in Fig. 1.

The matrix support was a $15 \mathrm{~mm} \times 40 \mathrm{~mm}$ copper plate, cooled below $20 \mathrm{~K}$ with a continuous flow of liquid helium. Ar gas was introduced into the chamber through a leak valve and the pressure set at $\sim 5 \times 10^{-6}$ Torr during the matrix condensation. The Ag deposition rate into the matrix was $0.05 \mathrm{~A} / \mathrm{s}$ (measured with a quartz crystal microbalance), corresponding to a metal loading of $0.8 \%$. The thickness of the matrix grown was around $100 \mathrm{~nm}$. The energy of the $\mathrm{Ar}^{+}$ion beam used to sputter the matrix was $2.5 \mathrm{keV}$, with current at the matrix of $3.2 \mu \mathrm{A}$. Minor of the ion beam fluctuations were addressed by setting the total charge deposited onto the cold finger constant $\left(2.2 \times 10^{-4} \mathrm{C}\right)$. The sputtering time (also the cluster deposition time) was $30 \mathrm{~s}$ for each sample. Referring to Fig. 1(b), it is possible to change the sputtering angle, $\alpha$, by rotating the matrix support. For each sputtering angle $(\alpha)$ employed, cluster were collected at a range of different angles $(\beta)$ by mounting different TEM grids on the same sample stage. The number and size distributions of the deposited clusters were obtained from high-angular annular dark field (HAADF) images in a probe-corrected STEM (JEOL 2100F, with a CEOS spherical aberration corrector). The HAADF images were acquired with inner and outer collection angles of 62 and $164 \mathrm{mrad}$ (camera length $10 \mathrm{~cm}$ ).

The molecular dynamics (MD) simulations performed to study the cluster emission process focused on the removal by sputtering of cluster already formed in the matrix (described as simulation Type S2 in our previous paper [26]). In the current work, a larger simulation cell (up to $432 \AA \times 432 \AA \times 320 \AA$ ) was constructed in order to simulate higher energy $(2 \mathrm{keV})$ ion impact. We embedded one Ag cluster of different sizes: 6,631, 1,965 or 321 atoms, with diameters of 6,4 or $2 \mathrm{~nm}$, respectively, in an amorphous Ar matrix. The total number of the atoms in the system is about 1.5 million. Unlike the previous simulations where the Ag cluster was embedded at a distance of $10 \AA$ below the open surface [26], here the cluster was only half-buried by the matrix, as shown in Fig. 2(a). The centre of the cluster is at $(0,0,0)$. The choice of the current simulation model is based on the benchmarking simulations as shown in the Electronic Supplementary Material (ESM). The cluster which is embedded at a distance of $2 \mathrm{~nm}$ below the open surface, had very limited chance to escape from the crater with a 45-degree impact and mainly remains half-buried on the surface. Therefore, we choose this well-defined model to study the effect of the energy deposition.

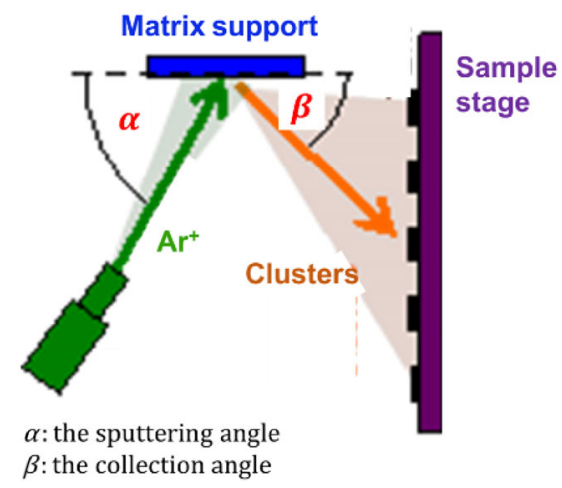

Figure 1 Definition of $\alpha$, the incident angle of $\mathrm{Ar}^{+}$beam, and $\beta$, the collection angle of the emitted clusters in the MACS. (a)

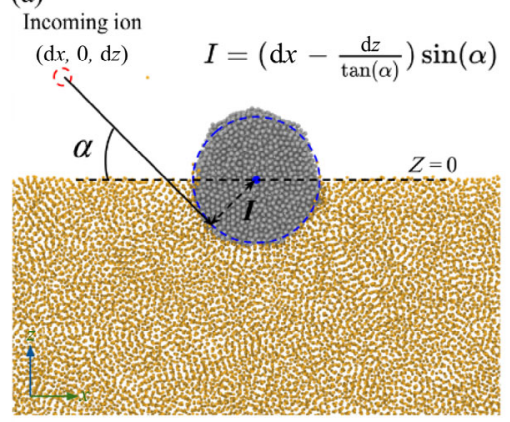

(b)

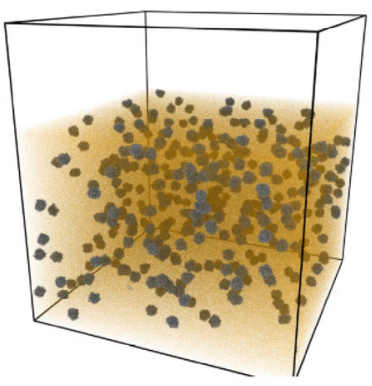

Figure 2 (a) Cross-sectional view of the initial configuration of the MD simulations. The Ag cluster atoms are grey and Ar matrix atoms orange. The red dashed circle indicates the initial location of the incoming $\operatorname{Ar}$ atom, $(\mathrm{d} x, 0, \mathrm{~d} z)$. The off-centre distance $I$ is the perpendicular distance from the centre of the cluster to the beam trajectory. (b) The initial configuration of the multi-irradiation simulation: 338 2-nm Ag clusters were randomly embedded in the Ar matrix.

We noticed that a single crystalline structure for the Ag cluster could lead to strong channelling effects [27] at certain incident angles. Therefore amorphous clusters were generated by the following process: (i) a quasi-spherical cluster was cut from the bulk fcc Ag, (ii) the cluster was heated to $2,000 \mathrm{~K}$ for $10 \mathrm{ps}$ and quenched to $10 \mathrm{~K}$ for 5 ps, so the cluster adopts a poly-crystalline structure, (iii) the cluster was rotated to a random axial angle, and (iv) the cluster was halfembedded in the Ar matrix and the interface relaxed to the local minimum energy to avoid overlap between Ag and Ar atoms. After the cluster was inserted, the simulation cell was additionally relaxed over $100 \mathrm{ps}$, so the initial system was in equilibrium at $10 \mathrm{~K}$. An incoming Ar atom was initially placed at $(\mathrm{d} x, 0, \mathrm{~d} z)$, where $\mathrm{d} x$ is the displacement in the $x$ direction and $\mathrm{d} z$ was equal to $50 \AA$ in all cases. We defined the off-centre distance $I$ as the perpendicular distance from the centre of the cluster to the ion beam. We ran the simulations with different values of $\mathrm{d} x$, ranging from -60 to $+60 \AA$ (the corresponding $I$ ranges from -42.4 to $42.4 \AA$ ). The neutral Ar atom is inserted instead of the positive charged ion, because Coulombic interaction between the ion and bulk matrix can cause the complex charge transfer which cannot be caught by classical MD. All simulations were conducted using the classical MD code LAMMPS [28] and results visualized using OVITO [29].

In order to directly compare with the experimental statistics, we also launched a multiple-impact irradiation simulation with an array of embedded clusters. As shown in Fig. 2(b), the initial structure consists of 338 2-nm Ag clusters which are randomly embedded in the matrix. The size of this cell was $432 \AA \times 432 \AA \times 320 \AA$. The corresponding Ag load was 4.7 at.\%. The structure was first prepared and relaxed following the same process as above. The Ar atoms with kinetic energies of $2 \mathrm{keV}$ were injected at random positions laterally from a starting point $8 \mathrm{~nm}$ above the original matrix surface. The incident angle, $\alpha$, was kept at $30^{\circ}$. During the simulation, all the sputtered atoms $150 \AA$ above the surface were removed from the simulation every 10,000 MD steps. We simulated 100 ion impacts and obtained 32 emission events.

\section{Results and discussion}

The yield and angular distribution of clusters emerging from the matrix for each four of incidence angles $\left(10^{\circ}, 15^{\circ}, 35^{\circ}\right.$ and $\left.45^{\circ}\right)$ were obtained from the cluster density in the STEM-HAADF images. Figure 3 shows a set of STEM-HAADF images, and corresponding size distribution graph for clusters prepared at $\alpha=10^{\circ}$ for three different collection angles. In each case, the size of the clusters spans the range from 50 to over 5,000 atoms/cluster and the peak size slightly shifts down as $\beta$ increases. However, this peak always lies between 200 and just over 300 atoms/cluster. 

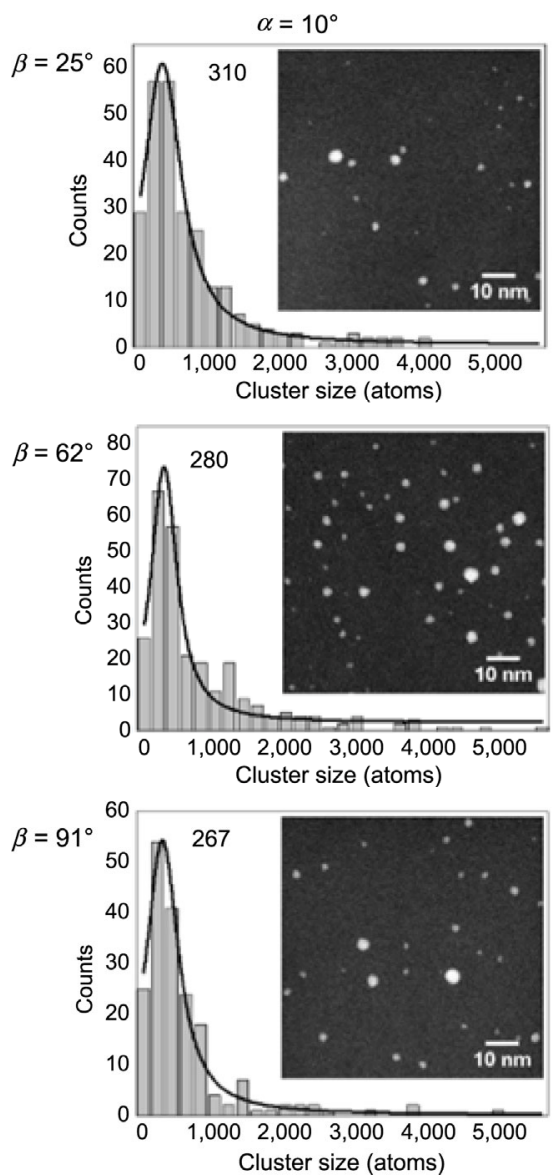

Figure 3 Examples of size distributions of clusters produced from the MACS matrix for a sputtering angle of $\alpha=10^{\circ}$ and different collection angle $\beta$.

Figure 4 shows STEM-HAADF images and corresponding size distributions for cluster prepared at three different sputtering angles, $\alpha$, of $10^{\circ}, 35^{\circ}$ and $45^{\circ}$, and integrated over the whole range of collection angles, $\beta$, ranging from $1^{\circ}$ to $120^{\circ}$. Again the peak size lies between 200 and 300 atoms/cluster. Most clusters (over 65\%) contain from 150 to 450 atoms/cluster. The results of Figs. 3 and 4 indicate that the cluster size distribution does not depend much at all on either the sputtering or collection angles.

In Fig. 5(a), the number of cluster collected as a function of the collection angle $\beta$ is reported for different sputtering angles $\alpha$. The overall yield of clusters increases for smaller $\alpha$ (more grazing incidence). The collection angle $\beta$ at which the highest intensity is measured, $\beta_{\max }$, decreases as $\alpha$ increases. As shown in Fig. 5(b), the relationship between $\alpha$ and $\beta_{\max }$ is in fact close to linear. Furthermore, the angle $\gamma$ subtended between the incident angle $\alpha$ and the corresponding $\beta_{\max }$, as calculated using Eq. (1), is approximately constant with a value between $99^{\circ}$ and $109^{\circ}$, as evidenced in Table 1 (the value decreases slightly with increased incident angle $\alpha$ ).

$$
\gamma=180-\left(\alpha+\beta_{\max }\right)
$$

From Figs. 5(a) and 5(b) it is possible to observe that the highest cluster flux is obtained when $\alpha=10^{\circ}$ and $\beta_{\max }=60^{\circ}$. We use MD simulations to shed some light on the atomistic origin of the observed angular dependence. In the benchmarking simulations, we study the crater formation in the pure Ar matrix under $2 \mathrm{keV}$ Ar irradiation as shown in Fig. S1 in the ESM. The result shows that the craters from 10 independent simulations, have the depth of $5 \mathrm{~nm}$ and the diameter of $10 \mathrm{~nm}$ in average. The further simulations with small Ag cluster embedded at the different depths $(0,-1,-2$ and $-3 \mathrm{~nm})$ indicate that the significant emission only occurs with shallowly embedded cluster (i.e., 0 and $-1-n m$ embedded depth). The deeply buried clusters mainly stay at the bottom of the crater as shown in
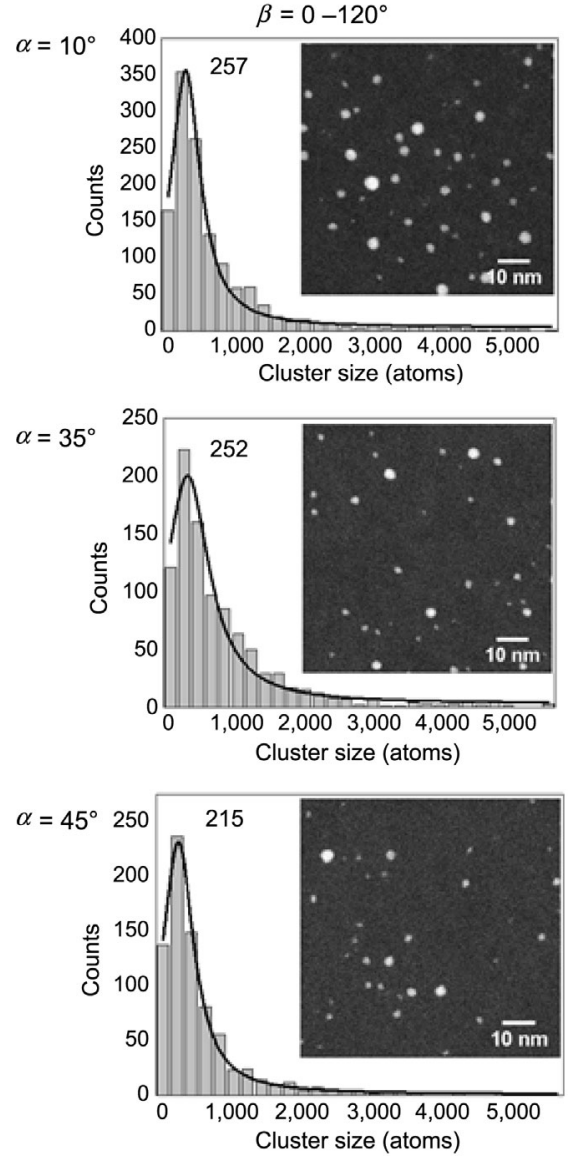

Figure 4 Examples of size distributions of clusters produced from the MACS matrix for different sputtering angles, $\alpha$, integrated over the collection angle, $\beta$.
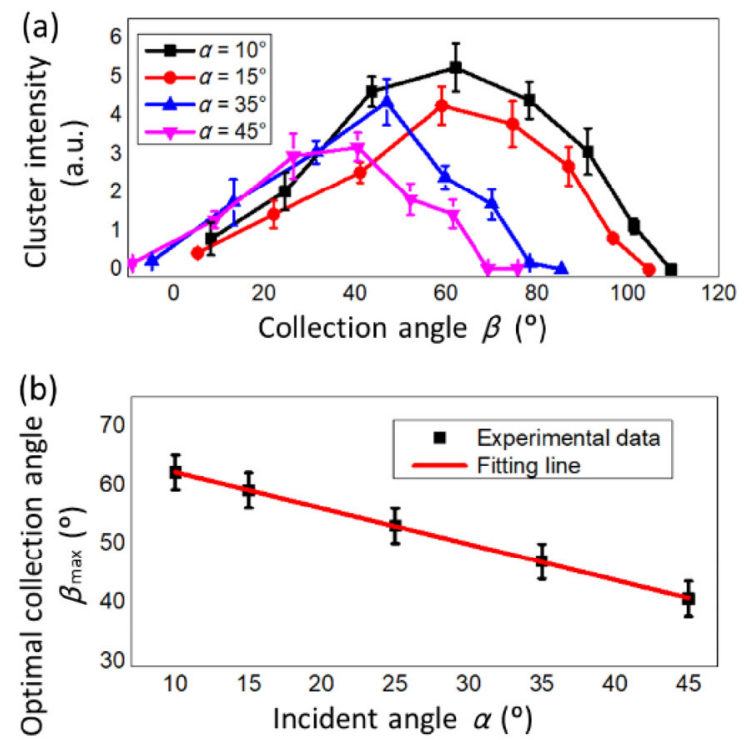

Figure 5 (a) Cluster intensity as a function of collection angle, $\beta$, for incident ion beam angle, $\alpha$, of $10^{\circ}, 15^{\circ}, 35^{\circ}$ and $45^{\circ}$. (b) Relationship between the optimal collection angle $\beta_{\max }$ and the incident angle $\alpha$.

Table 1 Angle $\gamma$ subtended between each $\alpha$ and the corresponding $\beta_{\max }$ as calculated from Eq. (1)

\begin{tabular}{ccc}
\hline$\alpha\left(^{\circ}\right)$ & $\beta_{\max }\left({ }^{\circ}\right)$ & $\gamma\left({ }^{\circ}\right)$ \\
\hline 10 & 61 & 109 \\
15 & 58 & 108 \\
35 & 43 & 102 \\
45 & 36 & 99 \\
\hline
\end{tabular}


Fig. S2 in the ESM. Therefore, we will focus on the emission of the half-buried clusters.

We identify two mechanisms which lead to any distribution we observe. If the Ar ion is stopped by the Ag cluster, as in case in Fig. 6(b), the deposited energy will be confined within the cluster before transfer to the Ar matrix across the whole matrix interface. This results in rather uniform sublimation of the matrix around the cluster, later leading on to nearly vertical cluster emission $\left(\beta \sim 90^{\circ}\right)$, as shown in Fig. 6(b6). In case of Fig. 6(a), the impact point is positively off the centre of the Ag cluster $(I=10.4 \AA)$, and the primary energy deposition is confined within the cluster, as shown in Fig. 6(a3). Therefore, the emission direction is again about vertical. By contrast, in case of Fig. 6(c), the negative off-centre impact causes the energy deposition to be focused on the other side of cluster/matrix interface, so the resultant collection angle $\beta$ is close to $60^{\circ}$. This result indicates that the primary energy deposition distribution must be an essential factor in determining the angular emission. Therefore, we further tested the effect of the initial impact point on the emission process. As shown in Fig. 6, the initial process of energy deposition is finished within the first 1 ps. The distribution of the deposited energy can be clearly mapped by examining the kinetic energies of atoms after about 1 ps, as shown in Fig. 6 in the third frame of each sequence. The full table is attached in Fig. S3 in the ESM, where more impact points are included. The dependence of the emission direction on the localized primary energy deposition is consistent with the selected results shown in Fig. 6.

In the schematic illustration of Fig. 7, we summarize four situations, depending on the impact point of the ion with respect to a $6 \mathrm{~nm} \mathrm{Ag}$ cluster; the orange colour intensity indicates the deposited energy trend. As shown in cases (a) and (d), if the impact point is far away from the cluster-matrix interface, the cluster will not detach from the matrix because of insufficient energy transfer to the nanocluster. In cases (b) and (c), the cluster and the interface can both stay in the high-temperature state for long enough to enable the emission process. In (b) the cluster is emitted on the opposite of the normal to the surface with respect to the incident ion. A key concept in (c) is the shadowing effect of the cluster for the incoming ion; a large fraction of ions with positive $I$ will be stopped within the cluster, without initiating any cascade in the Ar matrix in front of the cluster. The energy deposited from the ions in the cluster will be transmitted uniformly to the matrix and subsequently promote vertical cluster emission. Therefore, the average emission direction for $\alpha=45^{\circ}$ lies at $\beta=45^{\circ}-90^{\circ}$ for the $6 \mathrm{~nm}$ cluster. When we simulate the same process with smaller clusters of $4 \mathrm{~nm}$ in diameter, the results

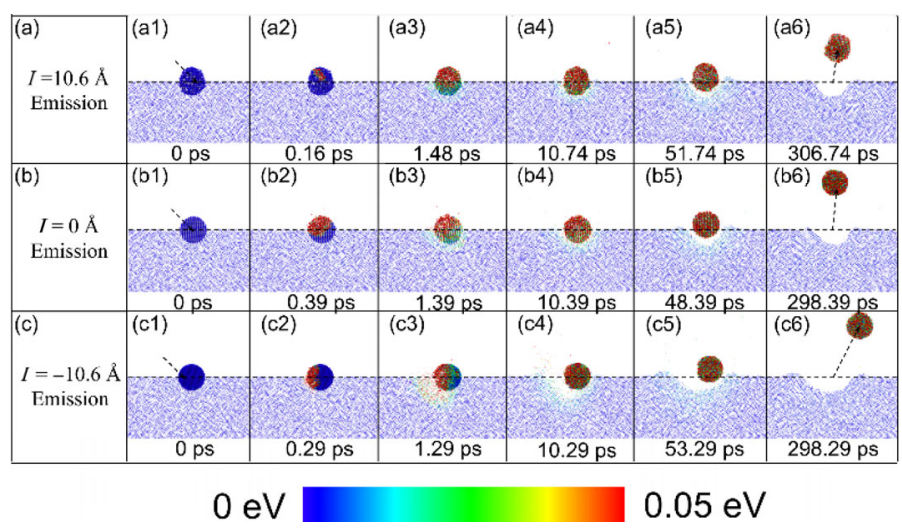

Figure 6 Two-dimensional cross-sectional views of the MD simulations. The Ag and $\mathrm{Ar}$ atoms are shown as large and small circles, respectively. The off-centre distance $I$ is equal to (a) $10.6 \AA$, (b) $0 \AA$ and (c) $-10.6 \AA$, respectively. The incident angle $\alpha$ is $45^{\circ}$ in all cases. The dashed lines in the first frame of each sequence show the incoming Ar ion. The dashed lines in the final frames show the emission direction of the Ag cluster. The colour coding shows the kinetic energy of the atom from 0 to $0.05 \mathrm{eV}$. A full table with $I$ range from -42.4 to $42.4 \AA$ is attached in Fig. S3 in the ESM.

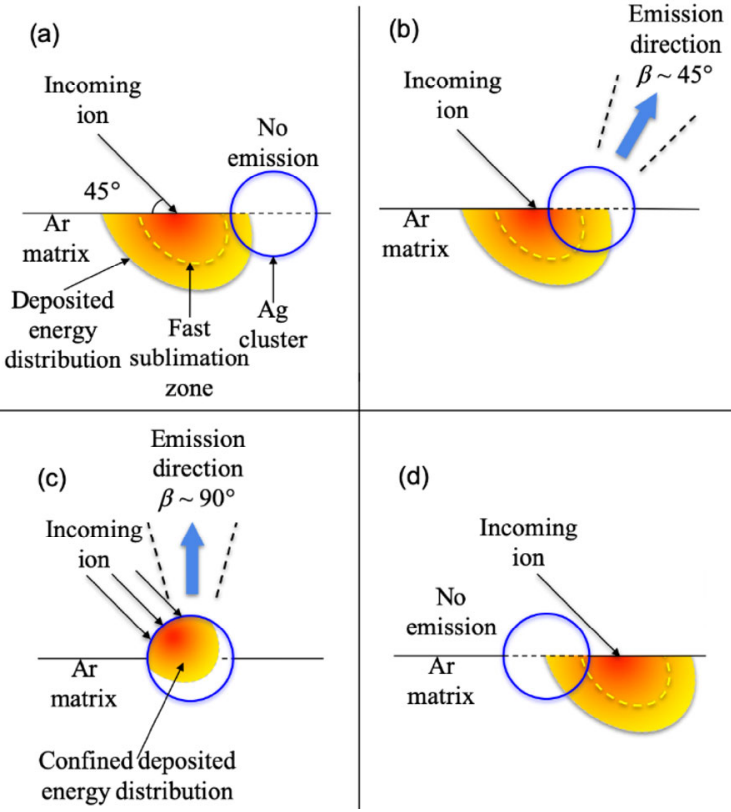

Figure 7 A schematic summary of the possible cases in the MD simulation. The incident angle $\alpha$ is $45^{\circ}$. (a) and (d) The impact point is far away from the cluster and no emission happens. (b) The impact point is close to the interface of the cluster and the matrix and causes the emission on the opposite side of the surface normal $\left(\beta=45^{\circ}-90^{\circ}\right)$. (c) The impact point is right on the cluster and the emission direction is vertical to the surface $\left(\beta \sim 90^{\circ}\right)$.

confirm that the primary location of the sublimation zone determines the angular dependence of the cluster emission. However, for 2-nm clusters (200-300 atoms/cluster) the energy confinement within the cluster is negligible. Therefore, these simulations struggle to explain the off-normal except for clusters larger than about $4 \mathrm{~nm}$, and we need to look for other explanations for the experimental distribution of Figs. 3 and 4. One factor that may merit consideration is the local surface roughness of the matrix, which is likely to affect the emission process (see Fig. S4(b) in the ESM). In fact, the matrix surface roughness increases from 0.85 to $35 \mathrm{~A}$ after only 100 ion impact events, as shown in Fig. S5 in the ESM.

In an attempt to better mimic the experimental conditions, we conducted another kind of simulation, by constructing a new matrix containing 338 randomly embedded clusters (rather than just one), which corresponds to $4.7 \%$ Ag loading in the solid Ar matrix. Although the metal loading is higher than the experimental value, the cascade and crater generated by $2 \mathrm{keV}$ Ar is within $5 \mathrm{~nm}$ in radius. Therefore, the well separated clusters can be considered as independent systems without interfering each other. The sizes of all the clusters are about $2 \mathrm{~nm}$ in diameter (190-220 atoms/cluster). A hundred successive $2 \mathrm{keV}$ irradiation events were simulated with the angle $\alpha$ set to $30^{\circ}$, which is representative of the experimentally investigated range. In these simulations, we observed 32 cluster emission events. As shown in Fig. 8, we identified these emission events as either "hit" or "miss", based on the value of the emission angle $\beta$ measured from the horizontal plane of the initial surface. If $\beta>90^{\circ}$ (red lines in Fig. 8(a)), the emitted cluster will "miss" the sample stage; if $\beta<90^{\circ}$ (green lines in Fig. $8(\mathrm{a})$ ), the cluster will likely be collected on the sample stage and counted in the statistics of the angular dependence of the emission. The histogram of the emission angles of all 19 "hits" events is shown in Fig. 8(b). The most probable emission angle, $\beta_{\max }$, from the simulations is around $50^{\circ}$, and the subtended angle between incident ion and $\beta_{\max }$ is thus $100^{\circ}$. These results are in good agreement with the experimental results; in effect, the surface roughness created by ion bombardment selects a privileged set of emitted trajectories for collection in the experiment.

Analysing in detail the dynamics of Ag cluster emission, we identified two kinds of cluster emission event. In the majority of 

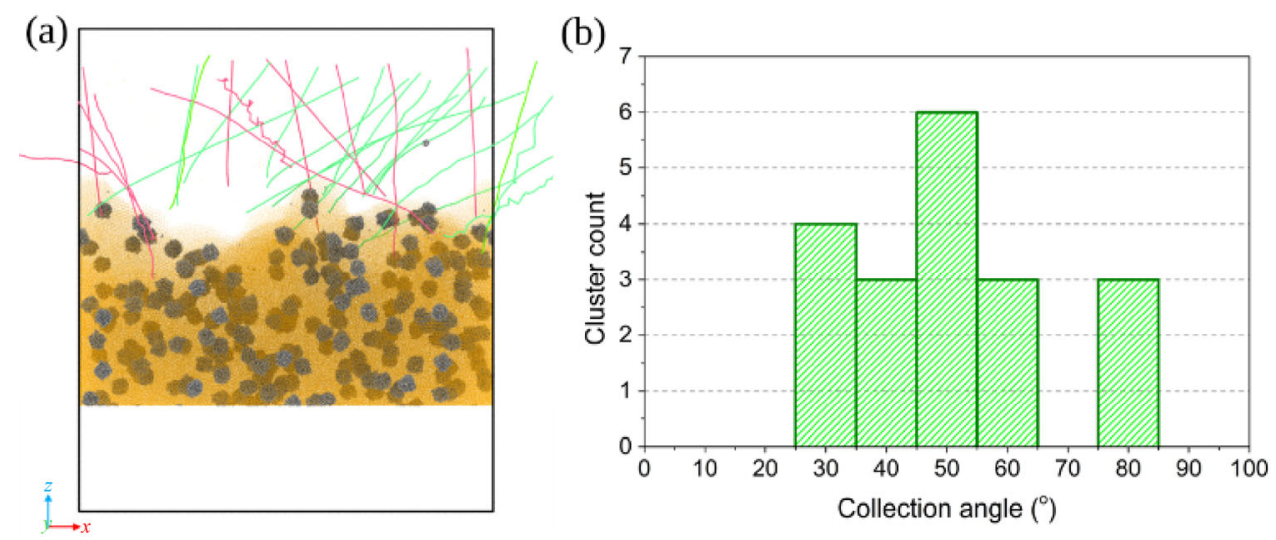

Figure 8 (a) The final configuration with illustrated trajectories of the emitted clusters (for the full movie, see Movie ESM1). The red trajectories indicate $\beta$ angles $>$ $90^{\circ}$, while the green trajectories indicate $\beta$ angles $<90^{\circ}$. (b) The histogram of cluster with $\beta$ angles $<90^{\circ}$. The most probable emission angle, $\beta_{\max }$, from the simulations is around $50^{\circ}$, and the subtended angle is $100^{\circ}$.

events, as shown in Fig. 9 Type I, the ion impacts in the vicinity of a Ag cluster and generates a collision cascade in the Ar matrix. The expansion of the heated cascade region generates a strong shock wave in the cold matrix; energy and momentum are transferred to the Ag cluster. If the cluster is close to the surface, and the cascade has initiated beneath it (more likely in grazing incidence), the shock wave is able to provide sufficient kick for the cluster to escape. On the other hand, a small fraction of the clusters receives a direct hit by the impacting ions, as shown in Fig. 9 Type II. In this case, a large fraction of the energy is deposited into these particles, dramatically raising their temperature, and there is no momentum transfer in favour of the emission at a specific angle. But the high temperature of the particle will heat up the surrounding Ar, possibly resulting in the random emission of the Ag cluster. The escaping clusters in Fig. 9, Type I and Type II, are indicated using dashed circular lines. Averaging over the different kinds of emission, the subtended angles are statistically biased towards $100^{\circ}$, which is independent of the incidence angle, as observed in the experiments (Fig. 5(a)), but is closely related to the shock wave formation and propagation. Moreover, due to the limits of the computational time required, the authors note that several experimental parameters, such as size distribution of the clusters and grazing angle emission rate, are not studied computationally in this paper. Further (Monte Carlo) simulations are necessary to comprehensively investigate these parameters, which is beyond the scope of the present study.

The measured cluster sizes range from tens of atoms to more than 5,000, and most of the collected clusters have a few hundred atoms, as shown in the Figs. 3 and 4; smaller clusters are less common as already discussed in our previous paper [24]. In this range of dimension (200-300 atoms/cluster) the simulations show that the cluster emission direction follows the thermal spike's path, described in [26]: clusters are emitted for $\beta$ both smaller and bigger than $90^{\circ}$. Because of the deposition system geometry, only clusters emitted for $\beta<90^{\circ}$ are collected on the sample stage (Fig. 1). Therefore, the formed shock wave causes the neighbouring clusters to be emitted with $\beta$ angles $<90^{\circ}$ and, as a consequence, the number of uncollected clusters decreases. Furthermore, as small cluster emission is guided by the Ar flow direction and thus by the formed shock wave propagation, the optimal emission direction subtends an angle of around $100^{\circ}$ with the incident direction, in harmony with the value obtained experimentally (as indicated in Table 1).

\section{Conclusions}

In this paper, we have reported the angular distribution of clusters generated in the MACS and shown that the highest cluster flux is obtained when the sputtering ion beam is near grazing $\left(\alpha=10^{\circ}\right)$. The measured cluster size was largely independent from MACS deposition system's geometry. We observed a correlation between the angle of incidence $\alpha$ and the collection angle with the highest cluster flux, $\beta_{\max }$. The angle subtended is approximately constant with value between $99^{\circ}$ and $109^{\circ}$.

(a) Type I

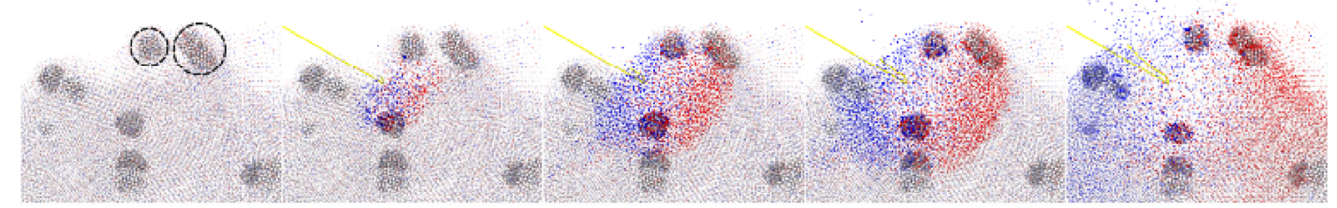

(b) Type II

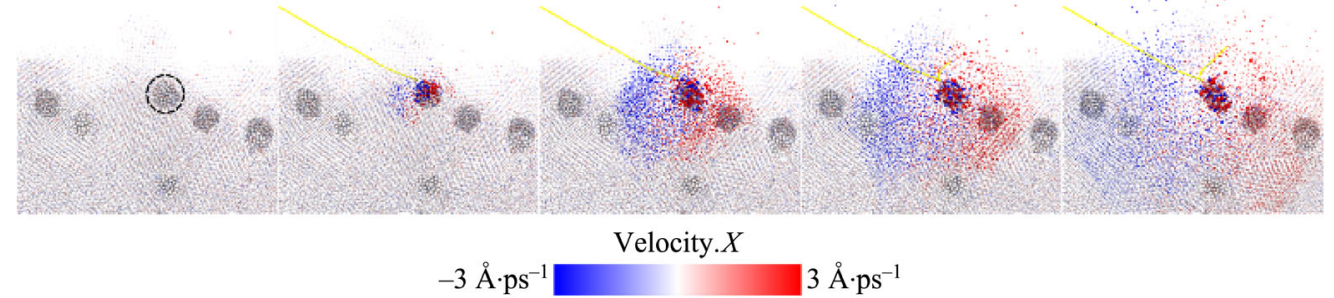

Figure 9 Two different impact processes from the continuous irradiation simulations. Type I: the ion impacts on the Ar matrix. The energy and momentum is transferred from the matrix to the cluster via the collective motion of the Ar atoms. Type II: the ion impacts on a cluster. The energy spreads from the cluster to the surrounding matrix. The colour coding represents the velocities of atoms along the $x$ axis. 
By MD simulations, we showed that direct ion impacts on a large metal nanocluster 4-6 $\mathrm{nm}$ in diameter lead to deposited energy confinement within the cluster and uniform matrix sublimation around it. Such nanoclusters emit mainly normally to the surface of the substrate. The non-uniform fast sublimation of the Ar matrix from one side of the cluster, on the other hand, will promote biased emission. These results suggest that the cluster size and the local surface configuration should regulate the emission of nanoclusters, since for the large size clusters the energy is mainly deposited within or near the cluster. The emission of small clusters $2 \mathrm{~nm}$ in diameter from a cryogenic matrix is shown to be driven by the shock waves which are generated by the collision cascades ignited by incoming ions beneath the near-surface nanoclusters. The direct comparison of MD simulations and the experiments shows a good measure of agreement between them. The MACS cluster emission mechanism investigated here should lead to more efficient cluster beam generation/collection, as required by industrial applications.

\section{Acknowledgements}

We thank the EPSRC, the Leverhulme Trust, Innovate UK and the EU H2020 project CritCat (project ID: 686053) for their financial support. Research performed at the University of Helsinki was supported by the EU project M4F (project ID: 755039). The authors are also grateful to the Finnish IT Centre of Science, CSC, for grants of computational time. The MACS concept is the subject of the following patent applications: Palmer, R. E.; PCT Patent Application No. WO2013017870; Europe Patent Application No. EP2739565; China Patent Application No. CN103857618; Japan Patent Application No. JP6316748; U.S. Patent Application No. US10233545.

Electronic Supplementary Material: Supplementary material (additional MD simulation and matrix surface roughness information) is available in the online version of this article at https://doi.org/ $10.1007 / \mathrm{s} 12274-019-2553-\mathrm{y}$.

Open Access This article is licensed under a Creative Commons Attribution 4.0 International License, which permits use, sharing, adaptation, distribution and reproduction in any medium or format, as long as you give appropriate credit to the original author(s) and the source, provide a link to the Creative Commons licence, and indicate if changes were made.

The images or other third party material in this article are included in the article's Creative Commons licence, unless indicated otherwise in a credit line to the material. If material is not included in the article's Creative Commons licence and your intended use is not permitted by statutory regulation or exceeds the permitted use, you will need to obtain permission directly from the copyright holder.

To view a copy of this licence, visit http://creativecommons.org/ licenses/by/4.0/.

\section{References}

[1] Knight, W. D.; Clemenger, K.; De Heer, W. A.; Saunders, W. A.; Chou, M. Y.; Cohen, M. L. Electronic shell structure and abundances of sodium clusters. Phys. Rev. Lett. 1984, 52, 2141-2143.

[2] Echt, O.; Sattler, K.; Recknagel, E. Magic numbers for sphere packings: Experimental verification in free xenon clusters. Phys. Rev. Lett. 1981, 47, $1121-1124$.

[3] Palmer, R. E.; Pratontep, S.; Boyen, H. G. Nanostructured surfaces from size-selected clusters. Nat. Mater. 2003, 2, 443-448.

[4] Palmer, R. E.; Cai, R. S.; Vernieres, J. Synthesis without solvents: The cluster (nanoparticle) beam route to catalysts and sensors. Acc. Chem. Res. 2018, 51, 2296-2304.

[5] Grammatikopoulos, P.; Steinhauer, S.; Vernieres, J.; Singh, V.; Sowwan, M. Nanoparticle design by gas-phase synthesis. Adv. Phys. X 2016, 1, 81-100.

[6] D'Addato, S.; Spadaro, M. C. Low pressure bottom-up synthesis of metal@oxide and oxide nanoparticles: Control of structure and functional properties. Phys. Scr. 2018, 93, 033001.
[7] Spadaro, M. C.; Luches, P.; Bertoni, G.; Grillo, V.; Turner, S.; Van Tendeloo, G.; Valeri, S.; D'Addato, S. Influence of defect distribution on the reducibility of $\mathrm{CeO}_{2-x}$ nanoparticles. Nanotechnology 2016, 27, 425705.

[8] Singh, A. V.; Vyas, V.; Patil, R.; Sharma, V.; Scopelliti, P. E.; Bongiorno, G.; Podestà, A.; Lenardi, C.; Gade, W. N.; Milani, P. Quantitative characterization of the influence of the nanoscale morphology of nanostructured surfaces on bacterial adhesion and biofilm formation. PLoS One 2011, 6, e25029.

[9] von Issendorff, B.; Palmer, R. E. A new high transmission infinite range mass selector for cluster and nanoparticle beams. Rev. Sci. Instrum. 1999, $70,4497-4501$.

[10] Skumryev, V.; Stoyanov, S.; Zhang, Y.; Hadjipanayis, G.; Givord, D.; Nogués, J. Beating the superparamagnetic limit with exchange bias. Nature 2003, $423,850-853$.

[11] Benelmekki, M.; Bohra, M.; Kim, J. H.; Diaz, R. E.; Vernieres, J.; Grammatikopoulos, P.; Sowwan, M. A facile single-step synthesis of ternary multicore magneto-plasmonic nanoparticles. Nanoscale 2014, 6, 3532-3535.

[12] D’Addato, S.; Pinotti, D.; Spadaro, M. C.; Paolicelli, G.; Grillo, V.; Valeri, S.; Pasquali, L.; Bergamini, L.; Corni, S. Influence of size, shape and core-shell interface on surface plasmon resonance in $\mathrm{Ag}$ and $\mathrm{Ag} @ \mathrm{MgO}$ nanoparticle films deposited on $\mathrm{Si} / \mathrm{SiO}_{x}$. Beilstein J. Nanotechnol. 2015, 6, 404-413.

[13] Pelli Cresi, J. S.; Spadaro, M. C.; D’Addato, S.; Valeri, S.; Benedetti, S.; Di Bona, A.; Catone, D.; Di Mario, L.; O'Keeffe, P.; Paladini, A. et al. Highly efficient plasmon-mediated electron injection into cerium oxide from embedded silver nanoparticles. Nanoscale 2019, 11, 10282-10291.

[14] Baxter, E. T.; Ha, M. A.; Cass, A. C.; Alexandrova, A. N.; Anderson, S. L. Ethylene dehydrogenation on $\mathrm{Pt}_{4,7,8}$ clusters on $\mathrm{Al}_{2} \mathrm{O}_{3}$ : Strong cluster size dependence linked to preferred catalyst morphologies. ACS Catal. 2017, 7,3322-3335.

[15] Carbone, R.; Giorgetti, L.; Zanardi, A.; Marangi, I.; Chierici, E.; Bongiorno, G.; Fiorentini, F.; Faretta, M.; Piseri, P.; Pelicci, P. G. et al. Retroviral microarray-based platform on nanostructured $\mathrm{TiO}_{2}$ for functional genomics and drug discovery. Biomaterials 2007, 28, 2244-2253.

[16] Pratontep, S.; Carroll, S. J.; Xirouchaki, C.; Streun, M.; Palmer, R. E. Size-selected cluster beam source based on radio frequency magnetron plasma sputtering and gas condensation. Rev. Sci. Instrum. 2005, 76, 045103.

[17] Wegner, K.; Piseri, P.; Tafreshi, H. V.; Milani, P. Cluster beam deposition: A tool for nanoscale science and technology. J. Phys. D Appl. Phys. 2006, 39, R439-R459.

[18] Llamosa, D.; Ruano, M.; Martinez, L.; Mayoral, A.; Roman, E.; GarciaHernández, M.; Huttel, Y. The ultimate step towards a tailored engineering of core@shell and core@shell@shell nanoparticles. Nanoscale 2014, 6, 13483-13486.

[19] Martínez, L.; Lauwaet, K.; Santoro, G.; Sobrado, J. M.; Peláez, R. J.; Herrero, V. J.; Tanarro, I.; Ellis, G. J.; Cernicharo, J.; Joblin, C. et al. Precisely controlled fabrication, manipulation and in-situ analysis of $\mathrm{Cu}$ based nanoparticles. Sci. Rep. 2018, 8, 7250.

[20] Kouznetsov, V.; Macák, K.; Schneider, J. M.; Helmersson, U.; Petrov, I. A novel pulsed magnetron sputter technique utilizing very high target power densities. Surf. Coat. Technol. 1999, 122, 290-293.

[21] Palmer, R. E.; Cao, L.; Yin, F. Note: Proof of principle of a new type of cluster beam source with potential for scale-up. Rev. Sci. Instrum. 2016, 87,046103

[22] Oiko, V. T. A.; Mathieu, T.; Cao, L.; Liu, J.; Palmer, R. E. Production of silver nanoclusters using a matrix-assembly cluster source with a solid $\mathrm{CO}_{2}$ matrix. J. Chem. Phys. 2016, 145, 166101.

[23] Ellis, P. R.; Brown, C. M.; Bishop, P. T.; Yin, J. L.; Cooke, K.; Terry, W. D.; Liu, J.; Yin, F.; Palmer, R. E. The cluster beam route to model catalysts and beyond. Faraday Discuss. 2016, 188, 39-56.

[24] Cai, R. S.; Jian, N.; Murphy, S.; Bauer, K.; Palmer, R. E. A new method to prepare colloids of size-controlled clusters from a matrix assembly cluster source. APL Mater. 2017, 5, 053405.

[25] Xu, J. Y.; Murphy, S.; Xiong, D. H.; Cai, R. S.; Wei, X. K.; Heggen, M.; Barborini, E.; Vinati, S.; Dunin-Borkowski, R. E.; Palmer, R. E. et al. Cluster beam deposition of ultrafine cobalt and ruthenium clusters for efficient and stable oxygen evolution reaction. ACS Appl. Energy Mater. 2018, 1, 3013-3018.

[26] Zhao, J. L.; Cao, L.; Palmer, R. E.; Nordlund, K.; Djurabekova, F. Formation and emission mechanisms of $\mathrm{Ag}$ nanoclusters in the Ar matrix assembly cluster source. Phys. Rev. Mater. 2017, 1, 066002.

[27] Nordlund, K.; Djurabekova, F.; Hobler, G. Large fraction of crystal directions leads to ion channeling. Phys. Rev. B 2016, 94, 214109.

[28] Plimpton, S. Fast parallel algorithms for short-range molecular dynamics. J. Comput. Phys. 1995, 117, 1-19

[29] Stukowski, A. Visualization and analysis of atomistic simulation data with OVITO-the open visualization tool modelling. Simul. Mater. Sci. Eng. 2010, 18, 015012. 